\title{
Ground state ferromagnetism in a generalized Hubbard model with strong correlations
}

\author{
L.Didukh; O.Kramar \\ Ternopil State Technical University, Department of Physics, \\ 56 Ruska Str., 46001 Ternopil, Ukraine
}

Received December 20, 2002

The present paper considers the ground state ferromagnetic ordering in a generalized Hubbard model with correlated hopping and interatomic exchange interaction in the case of strong correlations at non-integer band filling. The quasi-particle energy spectrum is obtained by means of a generalized mean-field approximation. The ground state energy of the system and the condition of ferromagnetic state realization are calculated. The dependences of magnetization on energy parameters and band-filling are obtained.

Key words: narrow energy band, ground state energy, ferromagnetism

PACS: 71.10.Fd, 75.10.-b, 75.20.En

\section{Introduction}

In recent years, intensive studies have been carried out concerning the electrical and the magnetic properties of the materials with a narrow energy band [1-3]. The narrow band model proposed by Hubbard [4] and its generalizations still attract great attention of the researchers (for review see references $[5,6]$ ). The Hubbard model is very rich in its physical contents in spite of its relative simplicity. In the framework of this model, an antiferromagnetic solution has been obtained, as well as a metal to insulator transition has been described. The conception of the Hubbard subbands is very useful in understanding the physical properties of narrow band materials, in particular, of the materials with high-temperature superconductivity. Nevertheless, there are a lot of problems in the physics of narrow energy bands, which are convenient to study in the framework of the Hubbard model and its generalizations but those problems have not yet been solved. One of them is the problem of ferromagnetism in a narrow energy band.

*E-mail: didukh@tu.edu.te.ua 
The Hubbard model with a partially filled band and the intra-atomic Coulomb repulsion energy $U \rightarrow \infty$ is the most favorable situation for ferromagnetism. In this case the antiferromagnetic exchange is suppressed and the effective Hamiltonian can be rewritten in the form (for band filling $n<1$ )

$$
H_{\mathrm{eff}}=\sum_{i j}^{\prime} t_{i j} c_{i \sigma}^{+} c_{j \sigma}
$$

where $c_{i \sigma}=\left(1-n_{i \bar{\sigma}}\right) a_{i \sigma}, a_{i \sigma}^{+}$and $a_{i \sigma}$ are the creation and destruction operators of electron on site $i, \sigma=\uparrow, \downarrow ; \bar{\sigma}$ denotes the spin projection which is opposite to $\sigma$, $n_{i \sigma}=a_{i \sigma}^{+} a_{i \sigma}$ is the operator of the number of electrons with spin $\sigma$ on site $i$. In spite of the large amount of papers where the Hamiltonian (1) has been studied, there is no decisive evidence for the ferromagnetism existence in such a system, though within some approximations (see below) the ferromagnetic solution has been obtained.

The Hamiltonian (1) yields the ferromagnetic solution already within Hubbard-I approximation [4] if the density of states with peculiarities is used. It is caused by the fact that the hopping of electrons in Hamiltonian (1) is described by quasi-fermi operators and ferromagnetism in the Hubbard model at $U \rightarrow \infty$ and $n \neq 1$ is the kinematic effect due to the non-fermi character of the commutation relations for the operators $c_{i \sigma}^{+}$and $c_{i \sigma}$ (it should be noted in the latter context that the "kinematic superconductivity" in the Hubbard model in some approximations [7] is realized).

For the model, described by Hamiltonian (1), the exact Nagaoka's result [8] is available; this result states that for the alternating lattices in the case of Coulomb repulsion $U \rightarrow \infty$ and exactly one electron above or below half-filling the ground state is ferromagnetic with a maximum total spin. The authors of reference [9] have generalized the Nagaoka's theorem on ferromagnetism in the Hubbard model (with one hole in a half-filled band) to the case where other nearest-neighbor Coulomb interactions (the interatomic Coulomb interaction, some type of correlated hopping, the exchange interaction and hopping of double occupancies) are included. The obtained results show that for non-zero interatomic exchange interactions $J$ the saturated ferromagnetic ground state is stable at finite $U>U_{\mathrm{c}}\left(U_{\mathrm{c}}\right.$ is a critical value of intra-atomic Coulomb repulsion dependent on the lattice type).

The study of ferromagnetism in the case of a finite number of holes in the framework of the approximations which improve the Hubbard-I approximation (see, for example [10-12]), leads to the conclusion that in the Hubbard model with a partially filled band (within some concentration interval) and with the strong Coulomb correlation, a ferromagnetic solution is possible. The important feature of the cited works (similar in the ideology to the work by Roth [13]) is the presence of a spin-dependent shift of the band centers; this is only a distinction [14] of the noted approximations from the Hubbard-I approximation. The developments of such approximations $[15,16]$ in the framework of modified perturbation theory [14] make it possible to obtain a ferromagnetic solution in the Hubbard model. The results of these considerations agree with the results of numerical finite-temperature MonteCarlo simulations solving the dynamical mean-field theory (DMFT) equations [17]. The authors of references [18,19] have obtained the ferromagnetic solution at in- 
termediate $U$-values and in a broad range of electron concentrations using a nonsymmetrical density of states with the peak near the band edge (in the framework of DMFT).

At the same time, in a series of papers the question concerning the existence of ferromagnetic solution in the Hubbard model (in particular, for the case described by Hamiltonian (1)) is disputed. It should be noted that the absence of ferromagnetism was proved in the Hubbard model in one-dimensional [20] and in two-dimensional [21] cases as well as in the case $n=1$ and at arbitrary finite $U$ [22]; in the latter context we also note the result of reference [23] where the absence of ferromagnetism has been stated in the Hubbard model at an arbitrary density of states. The ferromagnetic ordering is not realized in the case of small electron concentration $n \ll 1$ (the gas limit) [24] as well. The absence of ferromagnetism has been also stated in reference [25], where the calculation of magnetic susceptibility in the random phase approximation has been done. The extension of Nagaoka's theorem to the case of a system with a finite number of holes is not trivial whereas the thermodynamic limit for half-filling does not exist. Besides, in a series of papers [26-28] it has been emphasized that the increase of hole concentration destabilizes the ferromagnetic ordering. In particular, the results of reference [27] show that Nagaoka's state is not realized. It should be noted that approximations $[4,13,29,30]$ (and the papers cited above which follow the ideology of Roth) for the case of Mott-Hubbard insulator $(n=1$ and $U \rightarrow \infty)$ do not provide the Curie law for magnetic susceptibility [31]. It can be shown in the following way: if one generalizes Hamiltonian (1) by taking into account the interatomic exchange interaction in the mean-field approximation one obtains incorrect equations for magnetization and Curie temperature in the noted approximations. In particular, for the Curie temperature one obtains (in this connection see $[32,33])$ the expression

$$
\Theta_{\mathrm{C}}=\frac{w}{2 \operatorname{arcth}(w / z J)} .
$$

At $n=1$ and $U \rightarrow \infty$ the electrons are localized on the lattice sites and equation (2) has to take the mean-field form

$$
\Theta_{\mathrm{C}}=\frac{z J}{2}
$$

which can be obtained from (2) only if the unrealistic condition $w / z J \rightarrow 0$ is satisfied.

Although there are arguments which corroborate the existence of ferromagnetism in the Hubbard model, this problem requires further studies. In a series of papers [3437] the importance has been shown of generalization of the Hubbard model by taking into account other matrix elements of electron-electron interaction besides the intra-atomic Coulomb repulsion $U$, namely the correlated hopping and interatomic exchange interaction. In reference [19] it has been stated that taking into account above mentioned interactions the ferromagnetic state realization is considerably effected. Indeed, in a partially filled narrow energy band the translational part of the 
ground state energy $\sim n \delta w$ (here $\delta=1-n, w$ is the half-width of unperturbed band) and the exchange part of ground state energy $\sim z n^{2} J$. Close to half-filling $(n \rightarrow 1$, $\delta \rightarrow 0)$ the exchange part is crucial. Thereby, the consideration of interatomic exchange interaction is very important regardless of the possibility of ferromagnetic state occurrence due to the correlation shifts of the band centers. The importance of exchange interaction for the ferromagnetism realization has been also emphasized in references [9,38-41].

The distinctive feature of the Hubbard model, namely the electron-hole symmetry, is broken with taking into account the correlated hopping; this fact may be important forasmuch as the magnetic properties of transition metals and their compounds with less and with more than half-filled $3 d$-shell are asymmetrical. The consideration of correlated hopping within the mean-field approximation in the case of weak electron interactions shows $[37,40]$ that there are band narrowing and spindependent shifts of the band centers (due to correlated hopping) which favor the ferromagnetism realization. In the case of strong interactions, taking into account correlated hopping leads to renormalization of Hubbard bandwidth [34]. The effect of correlated hopping on the formation of ferromagnetic ordering has been also studied in reference [37] by the exact diagonalization of one-dimensional chains. The correlation effects (ferromagnetism and metal-insulator transition) connected with correlated hopping effect in the framework of the Gutzwiller method have been considered in reference [42] (the properties of the model in the limiting case of infinite space dimension $d \rightarrow \infty$ are analyzed). It has been obtained that correlated hopping favors the ferromagnetic ordering and lowers the critical value of intra-atomic Coulomb repulsion required for the occurrence of ferromagnetic spin ordering. At the same time it should be noted that the consideration of the correlated hopping effect of ferromagnetism within DMFT is non-trivial [42]. Therefore, the methods of linearization of Green function equations might be useful [5].

The present paper considers the model of narrow band material with electronhole asymmetry [43] for the study of ferromagnetism in narrow energy bands. In the case of strong electron correlation, the effective Hamiltonian is obtained. The treatment of the Hamiltonian is based on the use of a variant of second quantization method in the procedure of linearization of Green function equations. The efficiency of this approach has been approved earlier at the study of metal-insulator transition [44] at half-filling (in this case the obtained energy spectrum reproduces exact atomic and band limits, provides the correct behavior of ground state energy, energy gap and polar states concentration; at the basis of these results some peculiarities of narrow band materials have been interpreted).

\section{The model Hamiltonian}

We write the Hamiltonian of generalized Hubbard model in the form [43]:

$$
H=-\mu \sum_{i \sigma} a_{i \sigma}^{+} a_{i \sigma}+\sum_{i j \sigma}^{\prime}\left(t_{i j}+n T_{1}(i j)\right) a_{i \sigma}^{+} a_{j \sigma}+\sum_{i j \sigma}^{\prime}\left(T_{2}(i j) a_{i \sigma}^{+} a_{j \sigma} n_{i \bar{\sigma}}+\text { h.c. }\right)
$$




$$
+U \sum_{i} n_{i \uparrow} n_{i \downarrow}+\frac{J}{2} \sum_{i j \sigma \sigma^{\prime}}^{\prime} a_{i \sigma}^{+} a_{j \sigma^{\prime}}^{+} a_{i \sigma^{\prime}} a_{j \sigma},
$$

here $\mu$ is the chemical potential, $n=\left\langle n_{i \uparrow}+n_{i \downarrow}\right\rangle, t_{i j}$ is the matrix element which describes the hopping of electrons from site $j$ to site $i, T_{1}(i j)$ and $T_{2}(i j)$ are the integrals of correlated hopping of electrons, $U$ is the integral of intra-atomic Coulomb repulsion of electrons, $J$ is the integral of interatomic exchange interaction of electrons. The peculiarity of the model described by (4) is taking into account the effect of the site occupation on the hopping process (the correlated hopping).

The effect of correlated hopping of the first type $\left(T_{1}(i j)\right)$ is determined by occupancies of the neighboring sites which are not involved in the hopping process. This fact may be taken into account in the following way: we consider the presence of such occupancies by a peculiar mean-field which correlates the hopping of electrons. Therefore, the presence of correlated hopping leads to the concentration dependence of effective hopping integrals $t_{i j}(n)=t_{i j}+n T_{1}(i j)$. It should be noted that taking into account the correlated hopping of the first type $T_{1}(i j)$ in the mean-field approximation becomes exact in the homeopolar limit $n_{i \uparrow}+n_{i \downarrow}=1$. The peculiar feature of the correlated hopping of the second type $T_{2}(i j)$ is as follows: such a hopping of electrons occurs with the involvement of doubly occupied lattice sites. This fact leads to the electron-hole asymmetry in the model.

We write Hamiltonian (4) in the configurational representation using Hubbard transition operators $X_{i}^{k l}[29]$; the relation between the electron and X-operators is as follows:

$$
a_{i \sigma}^{+}=X_{i}^{\sigma 0}-\eta_{\sigma} X_{i}^{2 \bar{\sigma}}, \quad a_{i \sigma}=X_{i}^{0 \sigma}-\eta_{\sigma} X_{i}^{\bar{\sigma} 2},
$$

here $\eta_{\uparrow}=1, \eta_{\downarrow}=-1$.

In the case of strong Coulomb correlation $U \gg\left|t_{i j}\right|$, using the perturbation theory in the form proposed in reference [45] the effective Hamiltonian of generalized Hubbard model can be written in the form:

$$
H=H_{0}+H_{\mathrm{tr}}+H_{\mathrm{ex}}+\tilde{H}_{\mathrm{ex}},
$$

where

$$
\begin{aligned}
H_{0} & =-\mu \sum_{i}\left(X_{i}^{\uparrow}+X_{i}^{\downarrow}+2 X_{i}^{2}\right)+U \sum_{i} X_{i}^{2}, \\
H_{\mathrm{tr}} & =\sum_{i j \sigma}^{\prime} t_{i j}(n) X_{i}^{\sigma 0} X_{j}^{0 \sigma}+\sum_{i j \sigma}^{\prime} \tilde{t}_{i j}(n) X_{i}^{2 \sigma} X_{j}^{\sigma 2}, \\
H_{\mathrm{ex}} & =-\frac{1}{2} \sum_{i j \sigma} J_{i j}\left(\left(X_{i}^{\sigma}+X_{i}^{2}\right)\left(X_{j}^{\sigma}+X_{j}^{2}\right)+X_{i}^{\sigma \bar{\sigma}} X_{j}^{\bar{\sigma} \sigma}\right), \\
\tilde{H}_{\mathrm{ex}} & =-\frac{1}{2} \sum_{i j \sigma} \tilde{J}_{i j}\left(X_{i}^{\sigma} X_{j}^{\bar{\sigma}}-X_{i}^{\sigma \bar{\sigma}} X_{j}^{\bar{\sigma} \sigma}-X_{i}^{2} X_{i}^{0}\right),
\end{aligned}
$$

here

$$
\tilde{J}_{i j}=\frac{2\left(t_{i j}^{\prime}(n)\right)^{2}}{U}
$$


is the integral of indirect (through the polar states) exchange,

$$
\begin{aligned}
t(n) & =t\left(1-\tau_{1} n\right), \\
\tilde{t}(n) & =t\left(1-\tau_{1} n-2 \tau_{2}\right), \\
t^{\prime}(n) & =t\left(1-\tau_{1} n-\tau_{2}\right)
\end{aligned}
$$

are the hopping integrals in the lower Hubbard subband (hole subband), the upper Hubbard subband (doublon subband) and between these bands, respectively. For convenience the parameters of correlated hopping $\tau_{1}=T_{1} / t$ and $\tau_{2}=T_{2} / t$ are introduced, here $t$ is the band hopping integral for the nearest neighbors, $T_{1}$ and $T_{2}$ are the integrals of correlated hopping of the first and the second types for the nearest neighbors.

\section{Single-particle energy spectrum of narrow band model}

We use the retarded Green functions method [46] to obtain the single-particle energy spectrum. We introduce the Green function $G_{p p^{\prime}}(E)=\left\langle\left\langle X_{p}^{0 \sigma} \mid X_{p^{\prime}}^{\sigma 0}\right\rangle\right\rangle$ and write the equation of motion in the case $n<1$ when the processes with doublons can be neglected:

$$
\left(E+\mu+z J_{\mathrm{eff}} n_{\sigma}\right) G_{p p^{\prime}}(E)=\frac{\delta_{p p^{\prime}}}{2 \pi}\left\langle X_{p}^{\sigma}+X_{p}^{0}\right\rangle+\left\langle\left\langle\left[X_{p}^{0 \sigma}, H_{\mathrm{tr}}\right] \mid X_{p^{\prime}}^{\sigma 0}\right\rangle\right\rangle
$$

(in this case the exchange interaction is taken into account within the mean-field approximation, $z$ is the number of the nearest neighbor sites, $\left.J_{\text {eff }}=J-\tilde{J}\right)$. Similarly to reference [43] to obtain a self-consist equation for the Green function $G_{p p^{\prime}}(E)$ in accordance with the projection procedure we assume that

$$
\left[X_{p}^{0 \sigma}, H_{\mathrm{tr}}\right]=\sum_{j} \epsilon^{\sigma}(p j) X_{j}^{0 \sigma}
$$

where $\epsilon^{\sigma}(p j)$ is non-operator expression. After anticommutation of equation (16) with $X_{k}^{\sigma 0}$ we obtain:

$$
\begin{aligned}
\epsilon^{\sigma}(p k)\left(X_{k}^{\sigma}+X_{k}^{0}\right)= & t(n)\left(X_{p}^{\sigma}+X_{p}^{0}\right)\left(X_{k}^{\sigma}+X_{k}^{0}\right)+t(n) X_{k}^{\sigma \bar{\sigma}} X_{p}^{\bar{\sigma} \sigma} \\
& -\delta_{p k} t(n) \sum_{j} X_{k}^{\bar{\sigma} 0} X_{j}^{0 \bar{\sigma}}
\end{aligned}
$$

Then, equation (15) can be rewritten in the form:

$$
\begin{aligned}
\left(E+\mu+z J_{\mathrm{eff}} n_{\sigma}\right) G_{p p^{\prime}}(E)= & \frac{\delta_{p p^{\prime}}}{2 \pi}\left\langle X_{p}^{\sigma}+X_{p}^{0}\right\rangle+\sum_{j \neq p} \epsilon^{\sigma}(p j)\left\langle\left\langle X_{j}^{0 \sigma} \mid X_{p^{\prime}}^{\sigma 0}\right\rangle\right\rangle \\
& +\epsilon_{p}^{\sigma}\left\langle\left\langle X_{p}^{0 \sigma} \mid X_{p^{\prime}}^{\sigma 0}\right\rangle\right\rangle,
\end{aligned}
$$

The standard way of calculating the non-operator coefficients $\epsilon^{\sigma}(p j), \epsilon_{p}^{\sigma}$ is to average equation (17), that leads to the known approximations $[4,13,30]$. To obtain 
$\epsilon^{\sigma}(p j), \epsilon_{p}^{\sigma}$ we use a variant of the second quantization method. The relation between Hubbard operators and configurational operators has been found in reference [45]:

$$
X_{i}^{k l}=\alpha_{i k}^{+} \alpha_{i l}
$$

where $\alpha_{i k}^{+}, \alpha_{i l}$ are the creation and destruction operators of $|k\rangle$-state and $|l\rangle$-state on site $i$ (Shubin-Vonsowsky operators), respectively. For non-integer band-filling $n$ and magnetization $m$, in accordance with quasi-classical approximation, one can obtain:

$$
\begin{array}{rlrl}
\left\langle\alpha_{i \uparrow}^{+} \alpha_{i \uparrow}\right\rangle= & \frac{n-2 d+m}{2}, & \left\langle\alpha_{i \downarrow}^{+} \alpha_{i \downarrow}\right\rangle & =\frac{n-2 d-m}{2}, \\
\left\langle\alpha_{i 0}^{+} \alpha_{i 0}\right\rangle=c, & \left\langle\alpha_{i 2}^{+} \alpha_{i 2}\right\rangle=d .
\end{array}
$$

As in paper [43], substituting Hubbard operators by Shubin-Vonsowsky operators in equation(17)

$$
\begin{aligned}
& \alpha_{i \uparrow}^{+}=\alpha_{i \uparrow}=\left(\frac{n-2 d+m}{2}\right)^{\frac{1}{2}}, \quad \alpha_{i \downarrow}^{+}=\alpha_{i \downarrow}=\left(\frac{n-2 d-m}{2}\right)^{\frac{1}{2}}, \\
& \alpha_{i 0}^{+}=\alpha_{i 0}=(c)^{\frac{1}{2}}, \quad \alpha_{i 2}^{+}=\alpha_{i 2}=(d)^{\frac{1}{2}}
\end{aligned}
$$

we postulate a non-operator character of the expressions $\epsilon^{\sigma}(p j), \epsilon_{p}^{\sigma}$. Here, we introduce the notations

$$
\epsilon^{\sigma}(p j)=\alpha_{\sigma} t_{p j}(n), \quad \epsilon_{p}^{\sigma}=\beta_{\sigma}(p i) .
$$

After Fourier transformation of equation (18) we obtain the equation for Green function

$$
G_{\mathbf{k}}^{\sigma}=\frac{1}{2 \pi} \frac{1-n_{\bar{\sigma}}}{E-E_{\mathbf{k}}^{\sigma}}
$$

where single-particle energy spectrum $E_{\mathbf{k}}^{\sigma}$ has the form:

$$
E_{\mathbf{k}}^{\sigma}=-\mu+\alpha_{\sigma} t_{\mathbf{k}}(n)+\beta_{\sigma}-z J_{\text {eff }} n_{\sigma} .
$$

Here, the coefficient of correlation band-narrowing is

$$
\alpha_{\sigma}=1-n_{\bar{\sigma}}+\frac{n_{\bar{\sigma}} n_{\sigma}}{1-n_{\bar{\sigma}}}
$$

and the correlation shift of the band center is

$$
\beta_{\sigma}=\frac{w(n)(1-n) n_{\bar{\sigma}}}{1-n_{\bar{\sigma}}}
$$

$w(n)=z|t(n)|$ is the half-width of the lower subband, $n_{\sigma}$ is the concentration of electrons with spin $\sigma$ and it has been used such that

$$
t_{\mathbf{k}}(n)=\frac{1}{N} \sum_{i j} t_{i j}(n) \mathrm{e}^{\mathrm{i} \mathbf{k}\left(\mathbf{R}_{i}-\mathbf{R}_{j}\right)}=t(n) \gamma(\mathbf{k}), \quad \gamma(\mathbf{k})=\sum_{\mathbf{R}} \mathrm{e}^{\mathrm{i} \mathbf{k} \mathbf{R}}
$$


(the sum goes over the nearest neighbors to a site). Analogously, for the case $n>1$ we obtain

$$
\begin{aligned}
\tilde{G}_{\mathbf{k}}^{\sigma} & =\frac{1}{2 \pi} \frac{n_{\bar{\sigma}}}{E-\tilde{E}_{\mathbf{k}}^{\sigma}}, \\
\tilde{E}_{\mathbf{k}}^{\sigma} & =-\mu+\tilde{\alpha}_{\sigma} \tilde{t}_{\mathbf{k}}(n)+U+\tilde{\beta}_{\sigma}-z J_{\mathrm{eff}} n_{\sigma} .
\end{aligned}
$$

The expressions for $\tilde{\alpha}$ and $\tilde{\beta}$ are obtained from (25)-(26) by substituting $w(n)$ by $\tilde{w}(n)=z|\tilde{t}(n)|$ and $n$ by $2-n$.

The important peculiarity of the obtained spectra (24) and (29) is the presence of correlation narrowing of subbands, which has different widths due to correlated hopping. Besides, there is a spin-dependent shift of each subband center which favors the ferromagnetic state realization (see section 4).

\section{The ground state energy of the system}

Let us calculate the ground state energy of the system described by Hamiltonian (6). In the case $n<1$ averaging equation (6) we obtain

$$
\frac{E_{0}}{N}=\frac{1}{N} \sum_{i j \sigma} t_{i j}(n)\left\langle X_{i}^{\sigma 0} X_{j}^{0 \sigma}\right\rangle-z J_{\mathrm{eff}} n_{\uparrow}^{2}-z J_{\mathrm{eff}} n_{\downarrow}^{2},
$$

where the average in the last equation is determined by spectral intensity of Green function (23).

After the transition to quasi-impulse representation we integrate equation (30) using the density of states without peculiarities. In the case of rectangular density of states

$$
\rho(\epsilon)=\frac{1}{N} \sum_{\mathbf{k}} \delta(\epsilon-\epsilon(\mathbf{k}))=\frac{1}{2 w} \theta\left(\epsilon^{2}-w^{2}\right)
$$

for the ground state energy (per lattice site) we obtain

$$
\frac{E_{0}}{N}=\sum_{\sigma}\left(-\frac{(1-n) n_{\sigma}}{1-n_{\bar{\sigma}}} w(n)-z J_{\mathrm{eff}} n_{\sigma}^{2}\right) .
$$

The obtained expression can be rewritten in the form

$$
\frac{E_{0}}{N}=-\frac{(1-n)\left(2 n(2-n)-2 m^{2}\right)}{(2-n)^{2}-m^{2}} w(n)-\frac{z J_{\mathrm{eff}}}{2}\left(n^{2}+m^{2}\right)
$$

where $m$ is magnetization of the system.

In the case of semi-elliptic density of states

$$
\rho(\epsilon)=\frac{1}{N} \sum_{\mathbf{k}} \delta(E-\epsilon(\mathbf{k}))=\frac{2}{\pi w} \sqrt{1-\left(\frac{E}{w}\right)^{2}}
$$


one can obtain the following equations to calculate the ground state energy

$$
\begin{aligned}
& \frac{E_{0}}{N}=\sum_{\sigma}\left(-\frac{2\left(1-n_{\sigma}\right)}{3 \pi w(n)^{2}}\left(w(n)^{2}-x_{\sigma}^{2}\right)^{\frac{3}{2}}-z J_{\mathrm{eff}} n_{\sigma}^{2}\right) \\
& n_{\sigma}=\frac{\left(1-n_{\sigma}\right)}{\pi w(n)^{2}}\left(x_{\sigma} \sqrt{w(n)^{2}-x_{\sigma}^{2}}+w(n)^{2} \arcsin \left(x_{\sigma} w(n)\right)+w(n)^{2} \arcsin \left(w(n)^{2}\right)\right),
\end{aligned}
$$

here, $x_{\sigma}=\left(\mu-\beta_{\sigma}+z J_{\text {eff }}\right) / \alpha_{\sigma}$ (the results of numerical calculations are shown in figure 1).

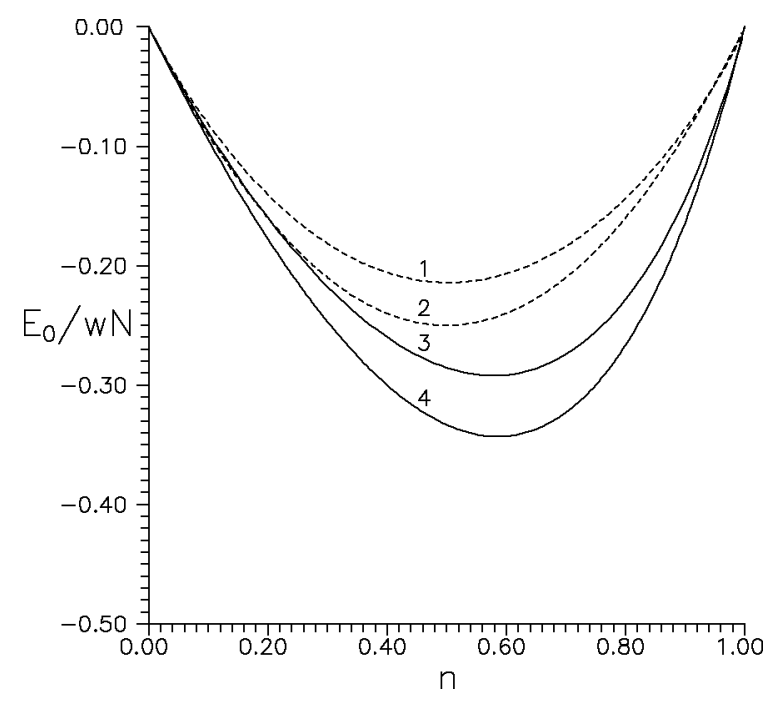

Figure 1. The concentration dependences of ground state energy of paramagnetic state (solid curves) and saturated ferromagnetic state (dotted curves): curves 1 and 3 correspond to the semielliptic density of states, curves 2 and 4 correspond to the rectangular density of states.

In figure 1 one can see that the ground state of the Hubbard model at $U \rightarrow \infty$ is paramagnetic and this conclusion is valid for a rectangular density of states (curves 2 and 4) as well as for semi-elliptic density of states (curves 1 and 3). Figure 2 shows the dependences of ground state energy on band-filling $n$ at different values of magnetization and fixed parameters of effective exchange and correlated hopping. The behavior of the ground state energy (at the same values of model parameters) indicates that in the range of concentrations close to half-filling, the ferromagnetic state is indeed realized. The correlated hopping substantially enlarges the range of electron concentration where the spontaneous magnetic moment exists.

To calculate the magnetization (this result and other ones are obtained for the rectangular density of states) we use the condition of ground state energy minimum $\mathrm{d} E_{0} / \mathrm{d} m=0$. The equation for equillibrium value of magnetization has the form (for the case of $n<1$ )

$$
\frac{8(1-n)^{2}(2-n)}{\left((2-n)^{2}-m^{2}\right)^{2}} m w(n)-m z J_{\mathrm{eff}}=0 .
$$

The criterion of ferromagnetism realization is obtained from the expression $\mathrm{d}^{2} E_{0} / \mathrm{d} m^{2}<0$

$$
z J_{\mathrm{eff}}>\frac{8(1-n)^{2}}{(2-n)^{3}} w(n)
$$

For the case $n>1$ one can obtain corresponding equations from equations (33)-(37) substituting $w(n)$ by $\tilde{w}(n)$ and $n$ by $2-n$. 
Using equation (36) one can get the critical values of the effective exchange integral which are required for the ferromagnetism realization. Figure 3 plots the dependence of $\left(z J_{\text {eff }} / w\right)$ on bandfilling at fixed values of magnetization (the case of generalized Hubbard model with exchange interaction). For the case of strong Coulomb interaction we obtain the correct behavior of critical exchange integral as a function of electron concentration $n$ (at distinct from the results of Hubbard-I approximation used in reference [41]). It should be noted that the range of band fillings, which is most favorable for ferromagnetism realization, is located close to half-filling. For the Hubbard model at half-filling the antiferromagnetic ordering is a distinctive feature. The competition of different types of magnetic ordering can be described by the parameter of effective

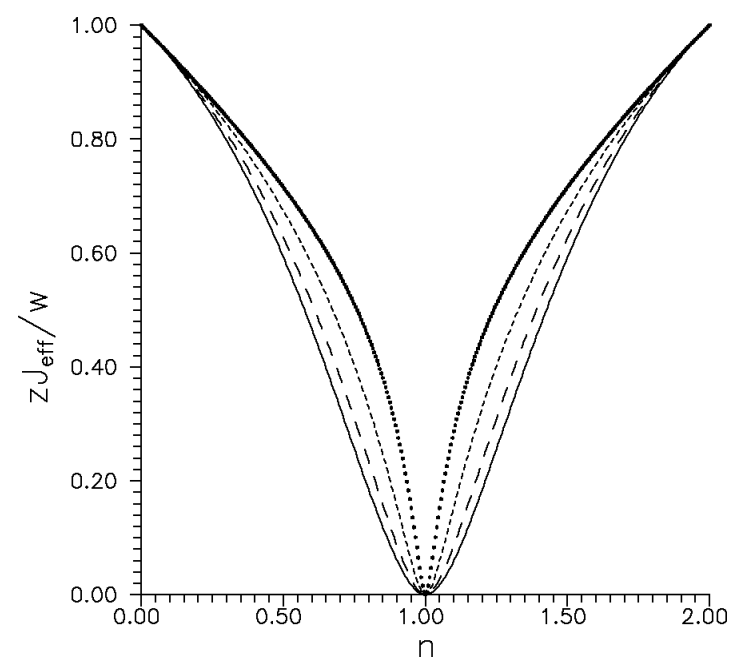

Figure 3. The critical values of an effective exchange integral $z J_{\text {eff }} / w$ as a function of band-filling $n$ at $\tau_{1}=\tau_{2}=0$. Curves (beginning from the lower one) correspond to the case $m=0, m=0.5 \cdot n, m=0.75 \cdot n$ and $m=n$, respectively.

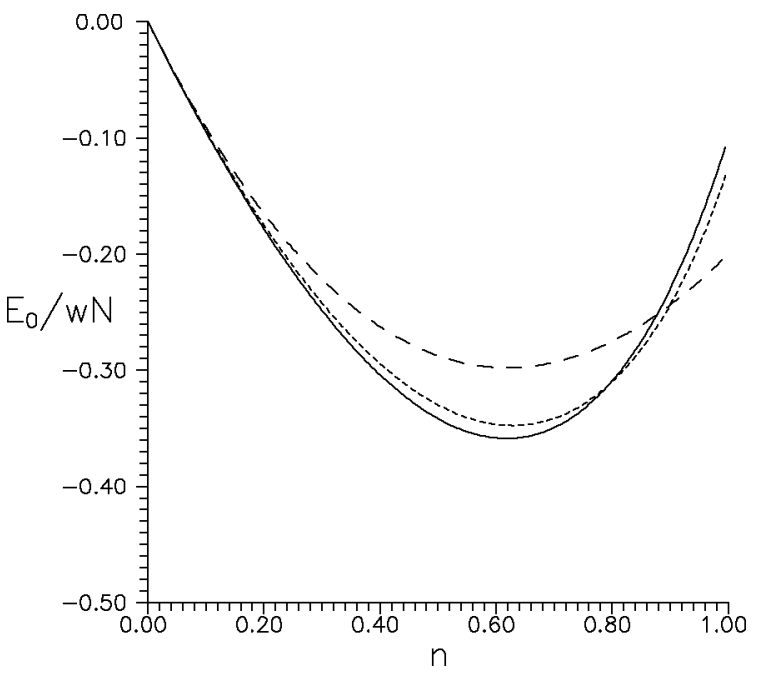

Figure 2. The concentration dependences of ground state energy of paramagnetic state (solid curve), ferromagnetic state ( $m=0.5 \cdot n$, dotted curve) and saturated ferromagnetic state (dashed curve) at $z J_{\text {eff }} / w=0.1, \tau_{1}=0.1, \tau_{2}=$ 0.4 .

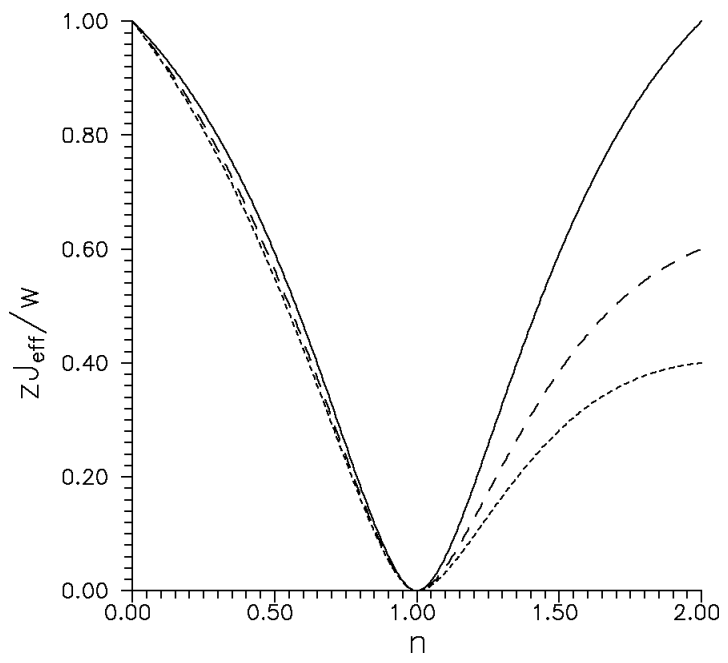

Figure 4. The concentration dependence of critical value of an effective exchange integral $z J_{\text {eff }} / w$. The upper curve corresponds to $\tau_{1}=\tau_{2}=0$; the middle curve corresponds to $\tau_{1}=$ $\tau_{2}=0.1$; the lower curve corresponds to $\tau_{1}=\tau_{2}=0.15$. 


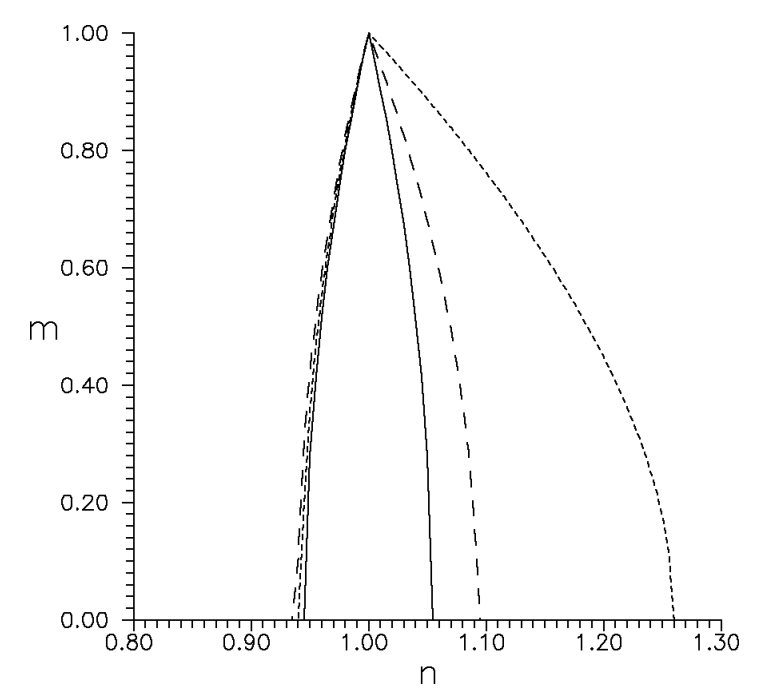

Figure 5. The concentration dependence of magnetization $m$ at $z J_{\text {eff }} / w=$ 0.02. The solid curve corresponds to $\tau_{1}=\tau_{2}=0$; the dashed curve corresponds to $\tau_{1}=\tau_{2}=0.2$; the dotted curve corresponds to $\tau_{1}=0.1 ; \tau_{2}=0.4$.

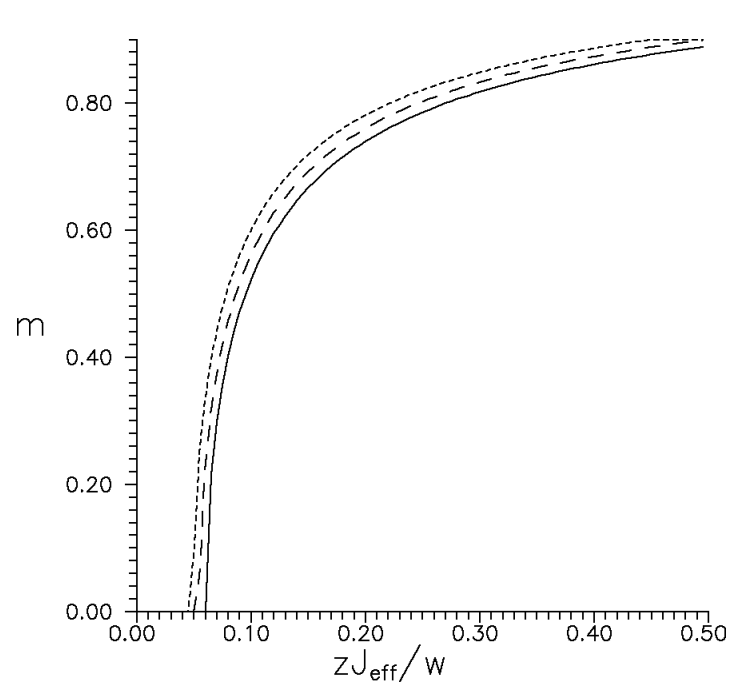

Figure 6. The dependence of magnetization $m$ on effective exchange interaction at $n=0.9$. The solid curve corresponds to $\tau_{1}=\tau_{2}=0$; the dashed curve corresponds to $\tau_{1}=\tau_{2}=0.1$; the dotted curve corresponds to $\tau_{1}=\tau_{2}=0.2$.

exchange interaction $J_{\text {eff }}$. The ferromagnetic ordering arises when $J_{\text {eff }}>0$; at the same time, if $2 t^{2} / U>J$, then the indirect antiferromagnetic exchange dominates (however, this problem in the present paper is not considered and will be studied elsewhere).

The effect of correlated hopping on the critical value of the effective exchange interaction is illustrated in figure 4 . The correlated hopping favors the occurrence of ferromagnetic ordering at non-integer band fillings $n$ : with the increase of the values of parameters $\tau_{1}$ and $\tau_{2}$ the critical value $z J_{\text {eff }} / w$ decreases. The case of electron concentration $n>1$ is more favorable for ferromagnetism which is in agreement with Hirsch's results [37]. In comparison with the case of weak Coulomb interactions [40], where more a favorable situation for ferromagnetism is determined by domination of some type of correlated hopping parameters, in the case of strong correlation, the crucial factor is the correlated hopping of the second type $\tau_{2}$ which comes out at $n>1$ (in this connection, note that ferromagnetic metals have more than a half-filled $3 d$-shell).

The magnetization of the system in the ground state obtained from equation (36) is plotted in figure 5 . The increase of electron concentrations (at $n<1$ ) in the band at fixed energy parameters of the model leads to the occurrence of a spontaneous magnetic moment; when the band filling reaches some critical value (at $n>1$ ) the magnetization vanishes. The peculiar feature of the plotted dependences is the enlargement of the concentration range where the ferromagnetic ordering is obtained caused by the effect of correlated hopping as well as the asymmetry with respect to half-filling which is characteristic of ferromagnetic materials. It should be also noted 
that the increase of an effective exchange interaction (at a fixed band-filling) leads to the increase of magnetization up to saturation values. Taking into account the correlated hopping makes it possible to obtain realistic critical values of an exchange integral (see figure 6).

\section{Conclusions}

The present paper studies the possibility of ferromagnetic state realization in the model of the material with strong Coulomb correlations. Taking into account the two types of the correlated hopping and direct exchange interaction is the peculiarity of the considered model.

In the strong correlation regime $U \gg|t|$ the single-particle energy spectrum is calculated at non-integer band-fillings $n$. The peculiar feature of the obtained energy spectrum is a narrowing of the band caused by electron correlation: the bandwidth depends on the electron concentration and on the correlated hopping parameters.

The calculated ground state energy depends on energy parameters of the system, on concentration of electrons $n$ and on magnetization $m$. From the expressions for the ground state energy, calculated using the density of states without peculiarities (rectangular and semi-elliptic), one can see that the exchange interaction is the crucial parameter for the stability of ferromagnetic ordering. The analysis of the condition of ferromagnetic ordering obtained from the ground state energy shows that both types of correlated hopping favor ferromagnetism and cause the asymmetry of the properties of the system with respect to the half-filling point. The case of more than half-filled band $n>1$ is more favorable for ferromagnetism realization than the opposite case $n<1$; from this point of view it is attractive to interpret the following fact: ferromagnetism of transitional metals and their alloys is realized in the system with more than half-filled $3 d$-shell. The concentration dependence of magnetization illustrates that the increase of the effective exchange interaction parameter (as well as correlated hopping parameters) causes the enlargement of the range of electron concentration where ferromagnetic ordering is realized. At some values of energy parameters of the system, the magnetization is saturated.

The results of this paper for the case of strong electron correlation supplements the results obtained in references [39,40], where the problem of ferromagnetism was studied in the case of weak and moderate electron interactions. The case of non-zero temperature and an application of the obtained results to explain of the properties of ferromagnetic materials will be considered in subsequent papers.

\section{References}

1. Baberschke K., Donath M., Nolting W. Band-Ferromagnetism. Ground-state and Finite-Temperature Phenomena. Berlin, Springer, 2001.

2. Abstracts of the International Conference on Strongly Correlated Electron Systems, Ann Arbor, Michigan, USA, 2001. 
3. Book of abstracts of International Conference on Strongly Correlated Electron Systems, Krakow, Poland, 2002.

4. Hubbard J. // Proc. Roy. Soc., 1963, vol. A276, p. 238.

5. Izyumov Yu. A. // Uspekhi Fizicheskikh Nauk, 1995, vol. 165, p. 403 (in Russian).

6. Fazekas P. Lecture Notes on Electron Correlation and Magnetism, Singapore, World Scientific Publishing, 1999.

7. Zajtsev R.O., Ivanov V.A. // Fiz. Tv. Tela, 1987, vol. 29, p. 3111 (in Russian).

8. Nagaoka Y. // Phys. Rev., 1966, vol. 147, p. 392.

9. Kollar M., Strack R., Vollhardt D. // Phys. Rev. B, 1996, vol. 53, No. 14. p. 9225.

10. Geipel G., Nolting W. // Phys. Rev. B, 1988, vol. 38, p. 2608; Nolting W., Borgiel W. // Phys. Rev. B, 1989, vol. 39, p. 6962.

11. Izyumov Yu.A., Letfulov B.M., Shipitsin E. V. // FMM, 1991, vol. 72, p. 90 (in Russian).

12. Goryachev E.G., Kuzmin E.V. // JETP, 1990, vol. 98, p. 1705 (in Russian).

13. Roth L.M. // Phys. Rev., 1969, vol. 184, p. 451.

14. Nolting W., Potthoff M., Herrmann T., Wegner T. - In: Band-Ferromagnetism. Ground-state and Finite-Temperature Phenomena. Berlin, Springer, 2001, p. 208.

15. Herrmann T., Nolting W. // J. Magn. Magn. Mater., 1997, vol. 170, p. 253.

16. Potthoff M., Herrmann T., Nolting W. // Phys. Stat. Sol. (b), 1997, vol. 210, p. 199.

17. Ulmke M. // Eur. Phys. J. B, 1998, vol. 1, p. 301.

18. Vollhardt D., Blümer N., Held K., Kollar M., Schlipf J., Ulmke M. // Z. Phys. B, 1997, vol. 103, p. 283.

19. Vollhardt D., Blümer N., Held K., Kollar M. - In: Band-Ferromagnetism. Groundstate and Finite-Temperature Phenomena. Berlin, Springer, 2001, p. 191.

20. Lieb E., Wu F. // Phys. Rev. Lett., 1968, vol. 20, p. 1445.

21. Rudin S., Mattis D. // Phys. Lett. A, 1985, vol. 110, p. 273.

22. Kemeny G. // Phys. Rev., 1967, vol. 24A, p. 307.

23. Fukuyama H., Ehrenreich H. // Phys. Rev. B., 1973., vol. 7, p. 3266.

24. Kanamori J. // Prog. Theor. Phys., 1963, vol. 30, No. 3, p. 275-289.

25. Kuzmin E.V. // Fiz. Tverd. Tela, 1997, vol. 39, p. 193 (in Russian).

26. Shastry B.S., Krishnamurthy H.R., Anderson P.W. // Phys. Rev. B, 1990, vol. 41, p. 2375 .

27. Izuyama T. // Progr. Theor. Phys., 1972, vol. 47, p. 2136-2137.

28. Kuzmin E.V. // Lett. to JETP, 1993, vol. 57, p. 575 (in Russian).

29. Hubbard J. // Proc. Roy. Soc. A., 1965, vol. 285, p. 542.

30. Harris A.B., Lange R.V. // Phys. Rev., 1967, vol. 157, p. 295.

31. Kawabata A. // Progr. Theor. Phys., 1972, vol. 48, p. 1793.

32. Kishore R., Joshi S. // Phys. Rev. B., 1979., vol. 2, p. 1411.

33. Didukh L. // Fiz. Met. i Metaloved., 1969, vol. 27, p. 1105 (in Russian).

34. Didukh L. // Fiz. Tverd. Tela, 1977, vol. 19, p. 1217 (in Russian).

35. Kivelson S., Su W.-P., Schrieffer J.R., Heeger A.J. // Phys. Rev. Lett., 1987, vol. 58, p. 1899.

36. Campbell D.K., Gammel J.T., Loh E.Y. // Phys. Rev. B, 1990, vol. 42, p. 475.

37. Amadon J.C., Hirsch J.E. // Phys. Rev. B, 1996, vol. 54, p. 6364.

38. Strack R., Vollhardt D. // Phys. Rev. Lett., 1994, vol. 72, p. 3425.

39. Hirsch J.E. // Phys. Rev. B, 1989, vol. 40, p. 2354; Hirsch J.E. // Phys. Rev. B, 1989, vol, 40, p. 9061. 
40. Didukh L., Kramar O., Skorenky Yu. // Phys. Stat. Sol. (b), 2002, vol. 229, p. 1241; Didukh L., Kramar O. // Low. Temp. Phys., 2002, vol. 28, p. 30.

41. Ivanov V. // Ukr. Phys. Journ., 1991, vol. 36, p. 751 (in Russian).

42. Kollar M, Vollhardt D. // Phys. Rev. B, 2001, vol. 63, p. 045107.

43. Didukh L. // Journ. of Phys. Stud., 1997, vol. 1, p. 241.

44. Didukh L., Hankevych V. // Phys. Stat. Sol. (b), 1999, vol. 211, p. 703; Didukh L., Hankevych V. // Condens. Matter Phys., 2001, vol. 4, No. 1(25), p. 93; Didukh L., Skorenky Yu. // Condens. Matter Phys., 2000, vol. 3, No. 4(24), p. 787.

45. Didukh L.D., Stasyuk I.V. // Ukr. Phys. Journ., 1968, vol. 13, p. 899.

46. Zubarev D.N. // Uspekhi Fizicheskikh Nauk, 1960, vol. 71, p. 71.

\section{Феромагнетизм в узагальненій моделі Габбарда 3 сильними кореляціями}

\section{Л.Дідух, О.Крамар}

Тернопільський державний технічний університет ім. І.Пулюя, кафедра фізики, 46001 Тернопіль, вул. Руська, 56

Отримано 20 грудня 2002 р.

В роботі розглядається основний феромагнітний стан для узагальненої моделі Габбарда з корельованим переносом та міжатомною обмінною взаємодією у випадку сильних електронних кореляцій та нецілого заповнення зони. 3 використанням узагальненого наближення середнього поля отримано квазічастинковий енергетичний спектр. Розраховано енергію основного стану та умову реалізації феромагнітного впорядкування. Отримано залежності намагніченості системи від значень енергетичних параметрів моделі та заповнення зони.

Ключові слова: вузька енергетична зона, енергія основного стану, феромагнетизм

PACS: $71.10 . F d, 75.10 .-b, 75.20 . E n$ 